\section{Evidence-based Dentistry: an Introduction}

\author{
Hackshaw AK, Paul EA, Davenport ES. Oxford:Blackwell
} Munksgaard. 2006 ISBN 1-4051-2496-2. Price $£ 27.50$

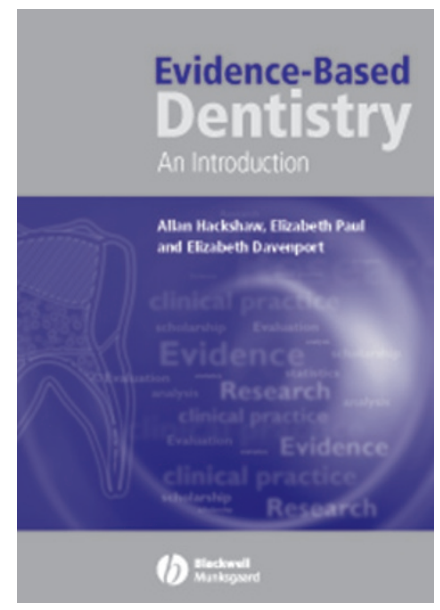

Very few textbooks are exclusively devoted to evidence-based dentistry (EBD), although a significant number of books have been written on topics related to evidence-based decision-making. This book has been designed as an introduction to understanding published research and its implications for the dental surgery. It is a groundlevel book for those seeking to understand EBD and its significance for clinical practice.

There are 10 chapters in total. The first summarises the purpose of EBD, followed by two chapters providing an introduction to some fundamental concepts, used later in the book. Chapter 4 shows how comparisons are made between groups of people, and chapters 2, 5, 6 and 7 cover the main types of study design, namely cross-sectional studies, randomised clinical trials, cohort studies and case-control studies. Next, chapter 8 compares and contrasts these different study designs and the following, penultimate, chapter discusses systematic reviews. The volume concludes by summarising important statistical concepts.

Throughout these sections, topical published papers from journals are used as worked examples for illustration and exercise. The papers chosen make the reader feel at home because the subjects are all relevant to the field of dentistry. Each paragraph of the paper is numbered to allow readers to easily pinpoint the particular section being discussed. Boxes are used extensively to present core information. Key points are summarised at the end of each chapter. These are followed by exercises with suggested answers.

The inclusion of eight published papers, some reprinted in full and some in part, demonstrates the authors' thoroughness and willingness to go to the extent of obtaining permission to reproduce these, in order to enable the readers to understand the relevant topics properly. Furthermore, grey tabs in the margin of these reprinted papers are designed to make them stand out clearly. It might be helpful to list these papers under the contents page as well.

Exercises at the end of a chapter are a good way for readers to utilise and apply the concepts covered by each. The section giving the authors' "suggested answers" at the back of the book has been delightfully laid out by placing alternate answers in shaded background. This gives a clear and easy-to-read design, further enhancing the user-friendly style of the book.

The concept of intention-to-treat analysis has been clearly illustrated using a hypothetical example, followed by an example taken from a dental journal. In the latter example, the analyses were reported to be intention-to-treat analyses, but they were in fact 'per protocol' analyses. The authors took the data given in the paper to recalculate the correct figures, and used this example as an illustration of the method for intention-to-treat analysis.

One quibble, however, is that although guidelines for the appraisal of clinical trials and observational studies are provided with examples in appendices, there is no mention of allocation concealment (which is different from blinding), nor the internationally recognised CONSORT (Consolidated Standards of Reporting Trials) statement.

The reading list at the end of the book, however, proves to be upto-date and useful for those wishing to pursue further about research methodology.

In summary, this book covers the basic knowledge and skills needed to begin to practise EBD. It will enable students and practitioners to gain the knowledge they require to assess a nd to utilise the increasing amount of research information available.

\section{Albert Yeung}

School of Dentistry, University of Manchester, Manchester, UK

Evidence-Based Dentistry (2007) 8, 24. doi:10.1038/sj.ebd.6400474 